\title{
Article \\ Comparison of Point-of-Care Testing and Hospital-Based Methods in Screening for Potential Type 2 Diabetes Mellitus and Abnormal Glucose Regulation in a Dental Setting
}

\author{
Muneedej Suwattipong ${ }^{1}$, Thitima Thuramonwong ${ }^{1}$, Chanita Tantipoj ${ }^{2}$, Pornpoj Fuangtharnthip ${ }^{2}$ (D), \\ Supanee Thanakun $^{3}$, Weerapan Khovidhunkit ${ }^{4}$ (D) and Siribang-on Piboonniyom Khovidhunkit ${ }^{2, *(D)}$ \\ 1 Dental Hospital, Faculty of Dentistry, Mahidol University, Bangkok 10400, Thailand; \\ muneedej.suw@mahidol.edu (M.S.); katae_katier@hotmail.com (T.T.) \\ 2 Department of Advanced General Dentistry, Faculty of Dentistry, Mahidol University, \\ Bangkok 10400, Thailand; ctantipoj@gmail.com (C.T.); pornpoj.fun@mahidol.ac.th (P.F.) \\ 3 College of Dental Medicine, Rangsit University, Muang Pathum Thani 12000, Thailand; \\ supanee.tha2@gmail.com \\ 4 Department of Medicine, Faculty of Medicine, Chulalongkorn University, Bangkok 10330, Thailand; \\ wkhovid@gmail.com \\ * Correspondence: siribangon.pib@mahidol.edu; Tel.: +66-2200-7853
}

check for updates

Citation: Suwattipong, M.;

Thuramonwong, T.; Tantipoj, C.; Fuangtharnthip, P.; Thanakun, S.; Khovidhunkit, W.; Khovidhunkit, S.P. Comparison of Point-of-Care Testing and Hospital-Based Methods in Screening for Potential Type 2 Diabetes Mellitus and Abnormal Glucose Regulation in a Dental Setting. Int. J. Environ. Res. Public Health 2021, 18, 6459. https:// doi.org/10.3390/ijerph18126459

Academic Editor: Paul B. Tchounwou

Received: 29 April 2021

Accepted: 11 June 2021

Published: 15 June 2021

Publisher's Note: MDPI stays neutral with regard to jurisdictional claims in published maps and institutional affiliations.

Copyright: (C) 2021 by the authors. Licensee MDPI, Basel, Switzerland. This article is an open access article distributed under the terms and conditions of the Creative Commons Attribution (CC BY) license (https:/ / creativecommons.org/licenses/by/ $4.0 /)$.

\begin{abstract}
This study aimed to compare the screening methods between point-of-care (POC) testing and hospital-based methods for potential type $2 \mathrm{DM}$ and abnormal glucose regulation (AGR) in a dental setting. A total of 274 consecutive subjects who attended the Faculty of Dentistry, Mahidol University, Bangkok, Thailand, were selected. Demographic data were collected. $\mathrm{Hb}_{1 \mathrm{c}}$ was assessed using a finger prick blood sample and analyzed with a point-of-care (POC) testing machine (DCA Vantage ${ }^{\circledR}$ ). Hyperglycemia was defined as POC $\mathrm{HbA}_{1 \mathrm{c}} \geq 5.7 \%$. Random blood glucose (RBG) was also evaluated using a glucometer (OneTouch ${ }^{\circledR}$ SelectSimple ${ }^{\mathrm{TM}}$ ) and hyperglycemia was defined as RBG $\geq 110 \mathrm{mg} / \mathrm{dl}$ or $\geq 140 \mathrm{mg} / \mathrm{dl}$. The subjects were then sent for laboratory measurements for fasting plasma glucose (FPG) and $\mathrm{HbA}_{1 \mathrm{c}}$. The prevalence of AGR (defined as FPG $\geq 100 \mathrm{mg} / \mathrm{dl}$ or laboratory $\mathrm{HbA}_{1 \mathrm{c}} \geq 5.7 \%$ ) and potential type $2 \mathrm{DM}$ (defined as FPG $\geq 126 \mathrm{mg} / \mathrm{dl}$ or laboratory $\mathrm{HbA}_{1 \mathrm{c}} \geq 6.5 \%$ ) among subjects was calculated and receiver operating characteristic (ROC) analysis was performed using FPG and $\mathrm{HbA}_{1 \mathrm{c}}$ for the diagnosis of AGR and potential type $2 \mathrm{DM}$. The prevalence of hyperglycemia defined as POC $\mathrm{HbA}_{1 \mathrm{c}} \geq 5.7 \%$, RBG $\geq 110 \mathrm{mg} / \mathrm{dl}$, and RBG $\geq 140 \mathrm{mg} / \mathrm{dl}$ was $49 \%, 63 \%$, and $32 \%$, respectively. After the evaluation using laboratory measurements, the prevalence of AGR was $25 \%$ and $17 \%$ using laboratory FPG and $\mathrm{HbA}_{1 \mathrm{c}}$ criteria, respectively. Based on the ROC curves, the performances of POC $\mathrm{HbA}_{1 \mathrm{c}}$ and RBG in predicting FPG-defined potential type 2 DM were high (AUC $=0.99 ; 95 \%$ CI $0.98-0.99$ and AUC $=0.94 ; 95 \%$ CI 0.86-1.0, respectively) but lower in predicting AGR (AUC $=0.72 ; 95 \%$ CI $0.67-0.78$ and AUC $=0.65 ; 95 \%$ CI 0.59-0.70, respectively). This study suggested that POC testing might be a potential tool for screening of subjects with potential type $2 \mathrm{DM}$ in a dental setting.
\end{abstract}

Keywords: point-of-care testing; diabetes mellitus; prevalence; dental clinics; hyperglycemia; abnormal glucose regulation

\section{Introduction}

Diabetes mellitus (DM) is a metabolic disease characterized by chronic hyperglycemia resulting from defects in insulin-producing cells, insulin action, or both [1]. The number of people aged $\geq 20$ years estimated to have type 2 DM globally is predicted to increase from 171 million in 2000 to 366 million by 2030 [2]. Undiagnosed type 2 DM are major problems encountered all over the world, and microvascular and macrovascular complications can possibly exist even in patients with prediabetes who had chronic hyperglycemia without any symptoms [2]. According to the data from the Thai National Health Examination 
Survey 2004, 2009, and 2014, the age-adjusted prevalence of DM reported in Thailand increased from $7.7 \%$ in 2004 to $7.8 \%$ in 2009 and $9.9 \%$ in 2014 (8.9\% among men and $10.8 \%$ among women). In addition, the proportion of undiagnosed DM remained high in 2014 (51.2\% for men and $41.3 \%$ for women) [3]. These facts support the urgent need to identify undiagnosed hyperglycemia and type $2 \mathrm{DM}$ earlier.

For decades, the diagnosis of DM has been based primarily on plasma glucose criteria, i.e., measurements of fasting plasma glucose (FPG) and plasma glucose after an oral glucose tolerance test (OGTT). Using American Diabetes Association (ADA) criteria, FPG level of less than $100 \mathrm{mg} / \mathrm{dl}$ is classified as normal, between $100-125 \mathrm{mg} / \mathrm{dl}$ is classified as prediabetes, and more than or equal to $126 \mathrm{mg} / \mathrm{dl}$ is classified as DM [4]. Despite being the diagnostic gold standard for DM, they are more time-consuming, labor-intensive, and impractical for DM screening since these gold standard methods need the patients to fast and cannot be performed after eating. After the discovery of glycated hemoglobin $\left(\mathrm{HbA}_{1 \mathrm{c}}\right)$, numerous studies have shown that $\mathrm{HbA}_{1 \mathrm{c}}$ could be used as an objective measurement of glycemic control [5]. In 2011, the World Health Organization (WHO) concluded that $\mathrm{HbA}_{1 \mathrm{c}}$ could be used as a diagnostic test for DM in accordance with strict quality assurance and test standardization [6]. According to the ADA, a $\mathrm{HbA}_{1 \mathrm{c}}$ level of less than $5.7 \%$ is considered to be normal, a $\mathrm{HbA}_{1 \mathrm{c}}$ between $5.7 \%$ and $6.4 \%$ is considered to be prediabetes, and a $\mathrm{HbA}_{1 \mathrm{c}}$ level greater than or equal to $6.5 \%$ is considered to be DM [4].

In addition to the hospital-based laboratory measurement of $\mathrm{HbA}_{1 c}$, point-of-care (POC) $\mathrm{HbA}_{1 \mathrm{c}}$ testing has also been used for screening of undiagnosed type $2 \mathrm{DM}$ in many healthcare settings. The use of POC $\mathrm{HbA}_{1 \mathrm{c}}$ as a screening or diagnostic tool has been reported in several studies [7-9]. For example, a population-based study conducted in 795 subjects aged $36-60$ years in a rural area in Uganda revealed that using POC $\mathrm{HbA}_{1 \mathrm{c}}$, $11.3 \%$ of subjects had DM compared with $4.8 \%$ for FPG [9]. With FPG as the reference, agreement between FPG and $\mathrm{HbA}_{1 \mathrm{c}}$ in classifying DM status was moderate (Kappa = 22.9; Area Under the Curve (AUC $=75 \%$ ), while that for abnormal glucose regulation (AGR) was low (Kappa $=11.0$; AUC $=59 \%$ ). However, agreement was high (over 90\%) among negative tests and among subjects with risk factors for type $2 \mathrm{DM}$, including obesity, overweight, and hypertension.

A random blood glucose (RBG) test is also used to screen and diagnose DM when hyperglycemic symptoms are present, along with the RBG level of $200 \mathrm{mg} / \mathrm{dl}$ or higher [1]. At present, blood glucose measurement using glucometers as a POC testing has been accepted worldwide for self-monitoring at home as well as for glucose monitoring in hospitalized patients [10].

Several studies have demonstrated that a dental setting could be a good venue for the diagnosis of people with undiagnosed hyperglycemia [11-13]. A systematic review regarding the screening for hyperglycemia in dental primary care practice settings was conducted [11]. High rates of undiagnosed hyperglycemia were detected among dental patients using POC testings. In our previous study, dental patients without a history of hyperglycemia were recruited and $\mathrm{HbA}_{1 \mathrm{c}}$ level was assessed using a finger prick blood sample and analyzed with the POC DCA Vantage ${ }^{\circledR}$ analyzer [12]. Of those 724 subjects, $33.8 \%$ had hyperglycemia defined as a POC $\mathrm{HbA}_{1 \mathrm{c}}$ level $\geq 5.7 \%$. In this $33.8 \%, 28.2 \%$ had prediabetes defined as a POC $\mathrm{HbA}_{1 \mathrm{c}}$ level of $5.7-6.4 \%$ and $5.6 \%$ had potential type $2 \mathrm{DM}$ defined as a POC $\mathrm{HbA}_{1 \mathrm{c}}$ level of $\geq 6.5 \%$. This prevalence was relatively high compared to the data from the NHES IV conducted among 18,629 Thai adults aged $\geq 20$ years, which found that the prevalence of impaired fasting glucose (IFG), defined as having an FPG level from $100-125 \mathrm{mg} / \mathrm{dl}$, and undiagnosed DM, defined as having an FPG level $\geq 126 \mathrm{mg} / \mathrm{dl}$, was $10.6 \%$ and $2.3 \%$, respectively [14].

Since there was no report of using POC $\mathrm{HbA}_{1 \mathrm{c}}$ and $\mathrm{RBG}$ for diagnosis and screening of potential type $2 \mathrm{DM}$ and AGR in Thailand, this study was conducted to investigate the use of POC $\mathrm{HbA}_{1 \mathrm{c}}$ and RBG in conjunction with symptoms of type $2 \mathrm{DM}$ to screen dental patients for AGR and type $2 \mathrm{DM}$ and to compare the accuracy of these POC measurements with the standardized hospital-based laboratory methods. 


\section{Materials and Methods}

\subsection{Study Population}

This clinical observational study was reviewed and approved by the Institutional Review Board, Faculty of Dentistry /Faculty of Pharmacy, Mahidol University (MU-DT/PY IRB 2017/047.2308). All subjects gave written informed consent prior to participation in the study. Inclusion criteria were patients aged between 20-70 years old who had no history of type $2 \mathrm{DM}$, needed emergency dental care, and attended the Primary and Emergency Unit of the Faculty of Dentistry, Mahidol University, Bangkok, Thailand. These consecutive patients that met the inclusion criteria were selected and required to fill in the demographic investigation form and a questionnaire. Exclusion criteria were severe anemia, polycythemia, secondary DM, pregnancy, or taking steroids, glucose-lowering medication, or chemotherapy. Patients who could not answer the questionnaire were also excluded. The flow of this study is presented in Figure 1.

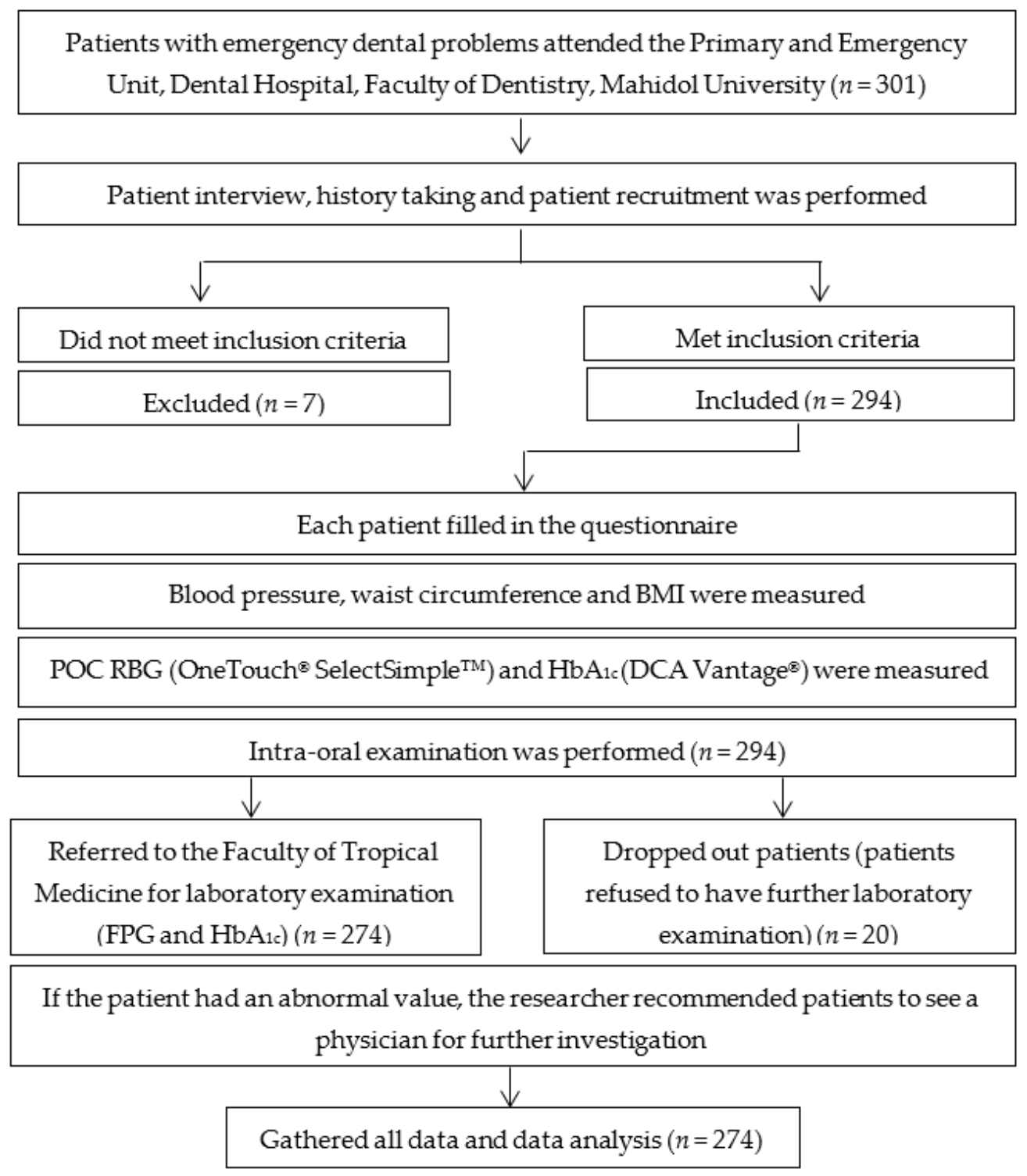

Figure 1. The flow of the research.

Sample size calculation was determined, using the estimate prevalence of hyperglycemia detected by RBG and $\mathrm{HbA}_{1 \mathrm{c}}$ and a formula for comparative two proportion [15]. At a significant level of $95 \%$, power of $80 \%$, estimated occurrence of hyperglycemia in dental setting being 30\% [12] and hypothesized difference in prevalence of hyperglycemia 
between the two tests at $17 \%$, the minimum computed sample size was 238 . This was adjusted to 274 respondents per group.

\subsection{Demographic Data Collection}

Demographic data collection was performed according to our previous study [12]. Briefly, a structured questionnaire was used to collect data regarding the patient's sex, age, marital status, type of work, smoking, alcohol consumption, and history of medical illness. Risk factors and symptoms of DM were also interviewed as the second part of the questionnaire. Symptoms of DM including polyuria, polydipsia, polyphagia, weight loss, blurred vision, paresthesia, taste disturbance, stress, bad breath, halitosis, and insomnia were also retrieved from each subject. Systolic and diastolic blood pressures were measured from the right arm in the seated position after the subject rested for at least $5 \mathrm{~min}$ using an automatic sphygmomanometer (Omron HEM-7221 ${ }^{\circledR}$, Omron Healthcare Co., Kyoto, Japan). Bodyweight was measured with a mechanical balance to the nearest $1.0 \mathrm{~kg}$. Standing height was measured and the body mass index (BMI) was calculated as weight $(\mathrm{kg})$ divided by height square $\left(\mathrm{m}^{2}\right)$. Overweight was defined as a BMI over $23 \mathrm{~kg} / \mathrm{m}^{2}$. Waist circumference $\geq 80 \mathrm{~cm}$ in females or $\geq 90 \mathrm{~cm}$ in males indicated central obesity [16].

\subsection{Periodontal Examination}

Study subjects received a full-mouth periodontal examination by 2 well-trained and experienced dentists (MS and TT). Periodontal examination was performed at the dental clinic using mouth mirrors and a manual periodontal probe (North Carolina periodontal probe UNC-15 Hu Friedy Manufacturing, Inc., Chicago, IL, USA) with an artificial dental unit light. Probing depth (PD) and recession were measured on all teeth except the third molar in 6 locations. The level of clinical attachment loss (CAL) was calculated from PD and recession, and it was represented as the distance from the cemento-enamel junction to the base of the periodontal pocket. Bleeding on probing (BOP) was recorded dichotomously as either present or absent. BOP was determined to be positive if hemorrhage occurred within $15 \mathrm{~s}$. Each participant was given instructions regarding dental treatment needs.

Subjects' periodontal status was classified into 3 levels, including (1) severe, (2) moderate, (3) mild or no periodontitis according to the extent and severity of periodontal disease using the following criteria: severe periodontitis ( $\geq 2$ interproximal sites with CAL $\geq 6 \mathrm{~mm}$ (not on the same tooth) and $\geq 1$ interproximal site with $\mathrm{PD} \geq 5 \mathrm{~mm}$ ), moderate periodontitis ( $\geq 2$ interproximal sites with CAL $\geq 4 \mathrm{~mm}$ (not on same tooth) and $\geq 2$ interproxmal site with $\mathrm{PD} \geq 5 \mathrm{~mm}$ ) and mild or no periodontitis (neither severe nor moderate periodontitis). In the questionnaire, the percentage of BOP site, percentage of the site with $\mathrm{PD} \geq 5 \mathrm{~mm}$, the number of missing teeth except for the third molar, and the total number of teeth that decayed, missed, and filled (DMF) were recorded.

\subsection{Glycemic Measurement}

For POC measurement, two blood drops were obtained from each participant, and each was placed on a separate applicator. The researcher performed a random capillary blood glucose (RBG) measurement with a portable blood glucose testing system $\left(\right.$ OneTouch $^{\circledR}$ SelectSimple ${ }^{\mathrm{TM}}$ blood glucometer test strips, LifeScan, Johnson and Johnson Inc., Charleston, SC, USA). The other drop of blood was used for the measurement of POC $\mathrm{HbA}_{1 \mathrm{c}}$. The DCA Vantage ${ }^{\circledR}$ analyzer (Siemens Medical Solutions Diagnostics, Tarrytown, $\mathrm{NY}$, USA), that quantitatively measured the percentage of $\mathrm{HbA}_{1 \mathrm{c}}$ in blood, based on latex agglutination inhibition immunoassay methodology, was used.

After initial POC RBG and POC $\mathrm{HbA}_{1 \mathrm{c}}$ were measured, all patients who had undergone POC testing were requested to come back on a convenient day (within 1 week) for a confirmation of their glycemic conditions using hospital-based laboratory methods including FPG and $\mathrm{HbA}_{1 \mathrm{c}}$ at the Faculty of Tropical Medicine, Mahidol University.

- Hyperglycemia. 
Hyperglycemia in this study was defined according to the POC testing, including RBG and POC $\mathrm{HbA}_{1 \mathrm{c}}$. We used 2 different cut-points of RBG at 110 and $140 \mathrm{mg} / \mathrm{dl}$ to indicate hyperglycemia, as previously reported in 2 separate studies, respectively $[17,18]$. POC hyperglycemia was also defined as a $\mathrm{HbA}_{1 \mathrm{c}} \geq 5.7 \%$ and this level was found to be very sensitive in identifying people with potential hyperglycemia [1].

- $\quad$ Potential type 2 DM.

Random blood glucose (RBG) test was also used to screen potential DM when DM symptoms are present along with the RBG level of $200 \mathrm{mg} / \mathrm{dl}$ or higher [1].

- $\quad$ AGR.

According to the ADA, an FPG level of less than $100 \mathrm{mg} / \mathrm{dl}$ was classified as normal, between $100-125 \mathrm{mg} / \mathrm{dl}$ was classified as prediabetes and more than or equal to $126 \mathrm{mg} / \mathrm{dl}$ was classified as DM [1]. In addition, for $\mathrm{HbA}_{1 \mathrm{c}}$, a level of less than $5.7 \%$ was considered to be normal, a $\mathrm{HbA}_{1 \mathrm{c}}$ between $5.7 \%$ and $6.4 \%$ was considered to be prediabetes, and a $\mathrm{HbA}_{1 \mathrm{c}}$ level greater than or equal to $6.5 \%$ was considered to be DM [1,4]. In our study, subjects with FPG $\geq 100 \mathrm{mg} / \mathrm{dl}$ or $\mathrm{HbA}_{1 \mathrm{c}} \geq 5.7 \%$ were classified as having AGR. Therefore, AGR in this study included both prediabetes and potential type $2 \mathrm{DM}$.

To convert the plasma glucose level from $\mathrm{mg} / \mathrm{dl}$ to $\mathrm{mmol} / \mathrm{L}$, the value of $\mathrm{mg} / \mathrm{dl}$ should be divided by 18 . For example, plasma glucose level of $110 \mathrm{mg} / \mathrm{dl}$ equals $110 / 18$ or $6.1 \mathrm{mmol} / \mathrm{L}$.

\subsection{Statistical Analysis}

All analyses were completed using SPSS, version 22.0 statistical software (SPSS Inc., Chicago, IL, USA). Sensitivity, specificity, positive predictive value (PPV) and negative predictive value (NPV) were compared between methods. Receiver operating characteristic (ROC) analysis was performed using hospital-based laboratory FPG and $\mathrm{HbA}_{1 \mathrm{c}}$ as gold standards for the diagnosis of AGR and potential type $2 \mathrm{DM}$.

\section{Results}

\subsection{Subjects' Characteristics}

A total of 274 consecutive subjects who attended the Primary and Emergency Unit of the Faculty of Dentistry, Mahidol University, with complete data, were included in this study. Characteristics of subjects are presented in Table 1 . Overall, $39 \%$ were male, and $61 \%$ were female. The mean age was $43 \pm 15$ years old. Approximately half of the subjects were younger than 40 years old ( $49 \%$ ), single, and had a BMI $\geq 23 \mathrm{~kg} / \mathrm{m}^{2}$. Over $60 \%$ of male and approximately half of the female subjects had central obesity. Most subjects were non-smokers, had no symptoms of type $2 \mathrm{DM}$, and had no family history of DM. Approximately $25 \%$ of subjects had high blood pressure, and $19 \%$ of subjects had severe periodontitis (Table 1).

Table 1. Subjects' characteristics distributed according to laboratory measurements.

\begin{tabular}{|c|c|c|c|c|c|}
\hline \multirow{2}{*}{ Characteristics } & \multirow{2}{*}{$\begin{array}{c}\text { Total } \\
n(\%)\end{array}$} & \multicolumn{2}{|c|}{ Laboratory $\mathbf{H b A}_{1 \mathrm{c}}$} & \multicolumn{2}{|c|}{ FPG } \\
\hline & & $<5.7 \% n(\%)$ & $\geq 5.7 \% n(\%)$ & $<100 \mathrm{mg} / \mathrm{dl} n(\%)$ & $\geq 100 \mathrm{mg} / \mathrm{dl} n(\%)$ \\
\hline \multicolumn{6}{|l|}{ Gender } \\
\hline Male & $106(38.7)$ & $80(75.5)$ & $26(24.5)$ & $71(67.0)$ & $35(33.0)$ \\
\hline Female & 168 (61.3) & $147(87.5)$ & $21(12.5)$ & $135(80.4)$ & 33 (19.6) \\
\hline \multicolumn{6}{|l|}{ Age } \\
\hline$\leq 40 y$ & $134(48.9)$ & $115(85.8)$ & $19(14.2)$ & $113(84.3)$ & $21(15.7)$ \\
\hline $41-60 y$ & $90(32.9)$ & $74(82.2)$ & $16(17.8)$ & $63(70.0)$ & $27(30.0)$ \\
\hline$\geq 61 \mathrm{y}$ & $50(18.3)$ & $38(76.0)$ & $12(24.0)$ & $30(60.0)$ & $20(40.0)$ \\
\hline \multicolumn{6}{|l|}{ Marital status } \\
\hline Single & 158 (57.7) & $133(84.2)$ & $25(15.8)$ & $114(72.2)$ & $44(27.8)$ \\
\hline Married & $77(28.1)$ & $63(81.8)$ & $14(18.2)$ & $60(77.9)$ & $17(22.1)$ \\
\hline Divorced/widow & $39(14.2)$ & $31(79.5)$ & $8(20.5)$ & $32(82.1)$ & $7(17.9)$ \\
\hline
\end{tabular}


Table 1. Cont.

\begin{tabular}{|c|c|c|c|c|c|}
\hline \multirow{2}{*}{ Characteristics } & \multirow{2}{*}{$\begin{array}{l}\text { Total } \\
n(\%)\end{array}$} & \multicolumn{2}{|c|}{ Laboratory $\mathrm{HbA}_{1 \mathrm{c}}$} & \multicolumn{2}{|c|}{ FPG } \\
\hline & & $<5.7 \% n(\%)$ & $\geq 5.7 \% n(\%)$ & $<100 \mathrm{mg} / \mathrm{dl} n(\%)$ & $\geq 100 \mathrm{mg} / \mathrm{dl} n(\%)$ \\
\hline \multicolumn{6}{|l|}{ Smoking status } \\
\hline Non-smoking & $203(74.1)$ & $175(86.2)$ & $28(13.8)$ & $158(77.8)$ & $45(22.2)$ \\
\hline Former smoking & $61(22.3)$ & $45(73.8)$ & $16(26.2)$ & $41(67.2)$ & $20(32.8)$ \\
\hline Current smoking & $10(3.7)$ & $7(70.0)$ & $3(30.0)$ & $7(70.0)$ & $3(30.0)$ \\
\hline \multicolumn{6}{|l|}{ Alcohol consumption } \\
\hline Never & $163(59.5$ & $139(85.3)$ & $24(14.7)$ & $124(76.1)$ & $39(23.9)$ \\
\hline Sometimes & $56(20.4)$ & $42(75.0)$ & $14(25.0)$ & $41(73.2)$ & $15(26.8)$ \\
\hline Usually & $55(20.1)$ & $46(83.6)$ & $9(16.4)$ & $41(74.5)$ & $14(25.5)$ \\
\hline \multicolumn{6}{|l|}{ Underlying disease } \\
\hline Yes & $193(70.4)$ & $166(86.0)$ & $27(14.0)$ & $149(72.2)$ & $44(22.8)$ \\
\hline No & $81(29.6)$ & $61(75.3)$ & $20(24.7)$ & $57(70.4)$ & $24(29.6)$ \\
\hline \multicolumn{6}{|l|}{ BMI } \\
\hline$\geq 23 \mathrm{~kg} / \mathrm{m}^{2}$ & $152(55.5)$ & $118(77.6)$ & $34(22.4)$ & $117(77.0)$ & $35(23.0)$ \\
\hline$<23 \mathrm{~kg} / \mathrm{m}^{2}$ & $122(44.5)$ & $109(89.3)$ & $13(10.7)$ & $89(73.0)$ & $33(27.0)$ \\
\hline \multicolumn{6}{|l|}{ Waist circumference } \\
\hline \multicolumn{6}{|l|}{ Male } \\
\hline$\geq 90 \mathrm{~cm}$ & $70(66.0)$ & $51(72.9)$ & $19(27.1)$ & $46(65.7)$ & $24(34.3)$ \\
\hline$<90 \mathrm{~cm}$ & $36(34.0)$ & $29(80.6)$ & $7(19.4)$ & $25(69.4)$ & $11(30.6)$ \\
\hline \multicolumn{6}{|l|}{ Female } \\
\hline$\geq 80 \mathrm{~cm}$ & $81(48.2)$ & $64(79.0)$ & $17(21.0)$ & $61(75.3)$ & $20(24.7)$ \\
\hline$<80 \mathrm{~cm}$ & $87(51.8)$ & $83(95.4)$ & $4(4.6)$ & $74(85.1)$ & $13(14.9)$ \\
\hline \multicolumn{6}{|l|}{ Family history of DM } \\
\hline Positive & $84(30.7)$ & $63(75.0)$ & $21(25.0)$ & $63(75.0)$ & $21(25.0)$ \\
\hline Negative & $190(69.3)$ & $164(86.3)$ & $26(13.7)$ & $143(75.3)$ & $47(24.7)$ \\
\hline \multicolumn{6}{|l|}{ Symptoms of DM } \\
\hline With at least 1 symptom & $44(16.1)$ & $33(75.0)$ & $11(25.0)$ & $31(70.5)$ & $13(29.5)$ \\
\hline Without any symptoms & $230(83.9)$ & $194(84.3)$ & $36(15.7)$ & $175(76.1)$ & $55(23.9)$ \\
\hline \multicolumn{6}{|l|}{ Hypertension } \\
\hline$\geq 140 / 90 \mathrm{mmHg}$ & $66(24.1)$ & $47(71.2)$ & $19(28.8)$ & $44(66.7)$ & $22(33.3)$ \\
\hline$<140 / 90 \mathrm{mmHg}$ & $208(75.9)$ & $180(86.5)$ & $28(13.5)$ & $162(77.9)$ & $46(22.1)$ \\
\hline \multicolumn{6}{|l|}{ Periodontal status } \\
\hline Mild/none & $173(63.1)$ & $156(90.2)$ & $17(9.8)$ & $149(86.1)$ & $24(13.9)$ \\
\hline Moderate & $48(17.5)$ & $36(75.0)$ & $12(25.0)$ & $30(62.5)$ & $18(37.5)$ \\
\hline Severe & $53(19.3)$ & $35(66.0)$ & $18(34.0)$ & $27(50.9)$ & $26(49.1)$ \\
\hline
\end{tabular}

FPG: fasting plasma glucose.

\subsection{Prevalence of Hyperglycemia and Potential Type 2 DM}

The prevalence of hyperglycemia defined as POC $\mathrm{HbA}_{1 \mathrm{c}} \geq 5.7 \%$ was $49 \%$. After the confirmation using the laboratory-based FPG and $\mathrm{HbA}_{1 \mathrm{c}}$, the prevalence of AGR was $25 \%$ and $17 \%$, respectively (Table 2). Regarding RBG, $37 \%, 56 \%$ and $7 \%$ had RBG $<110 \mathrm{mg} / \mathrm{dl}$, between $110-200 \mathrm{mg} / \mathrm{dl}$ and $>200 \mathrm{mg} / \mathrm{dl}$, respectively. After evaluation with FPG by the laboratory, $75 \%, 20 \%$ and $4 \%$ had FPG $<100 \mathrm{mg} / \mathrm{dl}$, between $100-125 \mathrm{mg} / \mathrm{dl}$ and $\geq 126 \mathrm{mg} / \mathrm{dl}$, respectively. When the laboratory $\mathrm{HbA}_{1 \mathrm{c}}$ was performed, $83 \%, 13 \%$ and $4 \%$ had $\mathrm{HbA}_{1 \mathrm{c}}<5.7 \%$, between $5.7-6.4 \%$ and $\geq 6.5 \%$, respectively (Table 2 ). Since another study suggested RBG level $\geq 140 \mathrm{mg} / \mathrm{dl}$ to increase the specificity of the screening for hyperglycemia in dental patients [18], the prevalence of hyperglycemia defined as RBG $\geq 140 \mathrm{mg} / \mathrm{dl}$ was also evaluated in this study. As shown in Table 2, the prevalence of hyperglycemia was reduced to $32 \%$. 
Table 2. Prevalence of hyperglycemia (POC $\mathrm{HbA}_{1 \mathrm{c}} \geq 5.7 \%$, POC RBG $\geq 110$ and $\geq 140 \mathrm{mg} / \mathrm{dl}$ ), prediabetes and potential type $2 \mathrm{DM}$ according to various methods of detection.

\begin{tabular}{cccc}
\hline Method & Range & Frequency & Percent \\
\hline \multirow{3}{*}{${\text { POC } \mathrm{HbA}_{1 \mathrm{c}}}$} & $<5.7 \%$ & 140 & 51 \\
& $5.7-6.4 \%$ & 115 & 42 \\
& $\geq 6.5 \%$ & 19 & 7 \\
& $\geq 5.7 \%$ & 134 & 49 \\
\hline \multirow{2}{*}{$\mathrm{RBG}$} & $<110 \mathrm{mg} / \mathrm{dl}$ & 101 & 37 \\
& $110-200 \mathrm{mg} / \mathrm{dl}$ & 154 & 56 \\
& $>200 \mathrm{mg} / \mathrm{dl}$ & 19 & 7 \\
& $\geq 110 \mathrm{mg} / \mathrm{dl}$ & 173 & 63 \\
\hline \multirow{2}{*}{$\mathrm{RBG}$} & $<140 \mathrm{mg} / \mathrm{dl}$ & 186 & 68 \\
& $140-200 \mathrm{mg} / \mathrm{dl}$ & 69 & 25 \\
& $>200 \mathrm{mg} / \mathrm{dl}$ & 19 & 7 \\
& $\geq 140 \mathrm{mg} / \mathrm{dl}$ & 88 & 75 \\
& $<100 \mathrm{mg} / \mathrm{dl}$ & 206 & 20 \\
& $100-125 \mathrm{mg} / \mathrm{dl}$ & 56 & 4 \\
FPG & $\geq 126 \mathrm{mg} / \mathrm{dl}$ & 12 & 25 \\
& $\geq 100 \mathrm{mg} / \mathrm{dl}$ & 68 & 83 \\
& $<5.7 \%$ & 227 & 13 \\
& $5.7-6.4 \%$ & 35 & 4 \\
\hline & $\geq 6.5 \%$ & 12 & 17 \\
\hline
\end{tabular}

POC: point-of-care; RBG: random blood glucose.

\subsection{Agreement between Hospital-Based Laboratory Measurement and POC Testing}

We next investigated the sensitivity, specificity, positive predictive values (PPV), and negative-predictive values (NPV) of the POC testing for AGR and potential type 2 DM using hospital-based laboratory measurements as gold standards (Tables 3 and 4). In Table 3, the hospital-based laboratory FPG levels of $\geq 100 \mathrm{mg} / \mathrm{dl}$ and $\geq 126 \mathrm{mg} / \mathrm{dl}$ were used as cut-off levels for AGR and potential type 2 DM, respectively. In Table 4 , the hospital-based laboratory $\mathrm{HbA}_{1 \mathrm{c}}$ levels of $\geq 5.7 \%$ and $\geq 6.5 \%$ were used as cut-off levels for AGR and potential type $2 \mathrm{DM}$, respectively.

Table 3. Sensitivity, specificity, PPV, and NPV of POC testing compared to hospital-based laboratory testing using FPG levels. $(n=274)$.

\begin{tabular}{|c|c|c|c|c|c|c|c|c|c|}
\hline \multirow[t]{2}{*}{ Testing } & \multicolumn{2}{|c|}{ Hospital-Based FPG Level } & & \multirow[t]{2}{*}{ Sens $* *$} & \multirow[t]{2}{*}{ Spec $* *$} & \multirow[t]{2}{*}{ PPV } & \multirow[t]{2}{*}{ NPV } & \multirow[t]{2}{*}{ AUC } & \multirow[t]{2}{*}{$95 \% \mathrm{CI}$} \\
\hline & Normal ${ }^{*} n(\%)$ & $\mathrm{AGR} * n(\%)$ & Total $n(\%)$ & & & & & & \\
\hline $\begin{array}{c}\text { POC } \mathbf{H b A}_{1 c} \\
<5.7 \% \\
\geq 5.7 \% \\
\text { Total }\end{array}$ & $\begin{array}{c}128(46.72) \\
78(28.47) \\
206(75.19)\end{array}$ & $\begin{array}{c}12(4.38) \\
56(20.44) \\
68(24.82)\end{array}$ & $\begin{array}{l}140(51.09) \\
134(48.91)\end{array}$ & 82.35 & 62.14 & 41.79 & 91.43 & 0.72 & $0.67-0.78$ \\
\hline $\begin{array}{c}\text { RBG cut-off }=\mathbf{1 1 0} \mathbf{~ m g / d l} \\
<110 \mathrm{mg} / \mathrm{dl} \\
\geq 110 \mathrm{mg} / \mathrm{dl} \\
\text { Total }\end{array}$ & $\begin{array}{c}91(33.21) \\
115(41.97) \\
206(75.19)\end{array}$ & $\begin{array}{c}10(3.65) \\
58(21.17) \\
68(24.82)\end{array}$ & $\begin{array}{l}101(36.86) \\
173(63.14)\end{array}$ & 85.29 & 44.17 & 33.53 & 90.10 & 0.65 & $0.59-0.70$ \\
\hline $\begin{array}{c}\text { RBG cut-off }=\mathbf{1 4 0} \mathbf{~ m g / d l} \\
<140 \mathrm{mg} / \mathrm{dl} \\
\geq 140 \mathrm{mg} / \mathrm{dl} \\
\text { Total }\end{array}$ & $\begin{array}{c}159(58.03) \\
47(17.15) \\
206(75.19)\end{array}$ & $\begin{array}{c}27(9.85) \\
41(14.96) \\
68(24.82)\end{array}$ & $\begin{array}{c}186(67.88) \\
88(32.12)\end{array}$ & 60.29 & 77.18 & 46.59 & 85.48 & 0.69 & $0.62-0.75$ \\
\hline
\end{tabular}


Table 3. Cont.

\begin{tabular}{|c|c|c|c|c|c|c|c|c|c|}
\hline \multirow[t]{2}{*}{ Testing } & \multicolumn{2}{|c|}{ Hospital-Based FPG Level } & \multirow[b]{2}{*}{ Total $n(\%)$} & \multirow[t]{2}{*}{ Sens $* *$} & \multirow[t]{2}{*}{ Spec $* *$} & \multirow[t]{2}{*}{ PPV } & \multirow[t]{2}{*}{ NPV } & \multirow[t]{2}{*}{ AUC } & \multirow[t]{2}{*}{$95 \% \mathrm{CI}$} \\
\hline & Normal * $n(\%)$ & $\mathrm{AGR} * n(\%)$ & & & & & & & \\
\hline & Non-DM * & Potential DM * & & & & & & & \\
\hline $\begin{array}{c}\text { POC } \mathbf{H b A}_{1 \mathrm{c}} \\
<6.5 \% \\
\geq 6.5 \% \\
\text { Total }\end{array}$ & $\begin{array}{c}255(93.07) \\
7(2.55) \\
262(95.62)\end{array}$ & $\begin{array}{c}0(0) \\
12(4.38) \\
12(4.38)\end{array}$ & $\begin{array}{c}255(93.07) \\
19(6.93)\end{array}$ & 100.00 & 97.33 & 63.16 & 100 & 0.99 & $0.98-0.99$ \\
\hline $\begin{array}{c}\text { RBG } \\
<200 \mathrm{mg} / \mathrm{dl} \\
\geq 200 \mathrm{mg} / \mathrm{dl} \\
\text { Total }\end{array}$ & $\begin{array}{c}254(92.70) \\
8(2.92) \\
255(93.07)\end{array}$ & $\begin{array}{c}1(0.36) \\
11(4.02) \\
12(4.38)\end{array}$ & $\begin{array}{c}255(93.07) \\
19(6.93)\end{array}$ & 91.67 & 96.95 & 57.89 & 99.61 & 0.94 & $0.86-1.00$ \\
\hline
\end{tabular}

* Normal indicates FPG levels < $100 \mathrm{mg} / \mathrm{dl}$; AGR indicates prediabetes and potential DM with FPG levels $\geq 100 \mathrm{mg} / \mathrm{dl}$; Non-DM indicates FPG levels < $126 \mathrm{mg} / \mathrm{dl}$; Potential DM indicates FPG levels $\geq 126 \mathrm{mg} / \mathrm{dl}$. ${ }^{* *}$ Sens: sensitivity; Spec: Specificity. PPV: positive predictive value; NPV: negative predictive value; AUC: area under the curve; DM: diabetes mellitus.

Table 4. Sensitivity, specificity, PPV, and NPV of POC testing compared to hospital-based laboratory testing using $\mathrm{HbA}_{1 \mathrm{c}}$ levels. $(n=274)$.

\begin{tabular}{|c|c|c|c|c|c|c|c|c|c|}
\hline \multirow[t]{2}{*}{ Testing } & \multicolumn{2}{|c|}{ Hospital-Based $\mathrm{HbA}_{1 \mathrm{c}}$ Level } & \multirow[b]{2}{*}{ Total $n(\%)$} & \multirow[t]{2}{*}{ Sens ** } & \multirow[t]{2}{*}{ Spec ** } & \multirow[t]{2}{*}{ PPV } & \multirow[t]{2}{*}{ NPV } & \multirow[t]{2}{*}{ AUC } & \multirow[t]{2}{*}{$95 \% \mathrm{CI}$} \\
\hline & Normal ${ }^{*} n(\%)$ & $\mathrm{AGR} * n(\%)$ & & & & & & & \\
\hline \multicolumn{10}{|l|}{ POC $\mathrm{HbA}_{1 \mathrm{c}}$} \\
\hline$<5.7 \%$ & $140(51.09)$ & $0(0)$ & $140(51.09)$ & 100 & 61.67 & 35.07 & 100 & 0.81 & $0.78-0.84$ \\
\hline$\geq 5.7 \%$ & $87(31.75)$ & $47(17.15)$ & $134(48.91)$ & & & & & & \\
\hline Total & $227(82.84)$ & 47 (17.15) & & & & & & & \\
\hline \multicolumn{10}{|c|}{ RBG cut-off $=110 \mathrm{mg} / \mathrm{dl}$} \\
\hline$<110 \mathrm{mg} / \mathrm{dl}$ & $94(34.31)$ & $7(2.55)$ & $101(36.86)$ & 85.11 & 41.41 & 23.12 & 93.07 & 0.63 & $0.57-0.69$ \\
\hline$\geq 110 \mathrm{mg} / \mathrm{dl}$ & $133(48.54)$ & $40(14.60)$ & $173(63.14)$ & & & & & & \\
\hline Total & $227(82.84)$ & 47 (17.15) & & & & & & & \\
\hline \multicolumn{10}{|c|}{ RBG cut-off $=140 \mathrm{mg} / \mathrm{dl}$} \\
\hline$<140 \mathrm{mg} / \mathrm{dl}$ & $169(61.68)$ & $17(6.20)$ & $186(67.88)$ & 63.83 & 74.45 & 34.09 & 90.86 & 0.69 & $0.62-0.77$ \\
\hline$\geq 140 \mathrm{mg} / \mathrm{dl}$ & $58(21.17)$ & $30(10.95)$ & $88(32.12)$ & & & & & & \\
\hline \multirow[t]{2}{*}{ Total } & $227(82.84)$ & 47 (17.15) & & & & & & & \\
\hline & Non-DM * & Potential DM * & & & & & & & \\
\hline \multicolumn{10}{|l|}{$\mathrm{POC} \mathbf{H b}_{1 \mathrm{c}}$} \\
\hline$<6.5 \%$ & $255(93.07)$ & $0(0)$ & $255(93.07)$ & 100 & 97.33 & 63.16 & 100 & 0.99 & $0.98-0.99$ \\
\hline$\geq 6.5 \%$ & $7(2.55)$ & $12(4.38)$ & $19(6.93)$ & & & & & & \\
\hline Total & $262(95.62)$ & $12(4.38)$ & & & & & & & \\
\hline \multicolumn{10}{|l|}{ RBG } \\
\hline$<200 \mathrm{mg} / \mathrm{dl}$ & $253(92.34)$ & $2(0.73)$ & 255 (93.07) & 83.33 & 96.56 & 52.63 & 99.22 & 0.90 & $0.79-1.00$ \\
\hline$\geq 200 \mathrm{mg} / \mathrm{dl}$ & $9(3.28)$ & $10(3.65)$ & 19 (6.93) & & & & & & \\
\hline Total & $262(95.62)$ & $12(4.38)$ & & & & & & & \\
\hline
\end{tabular}

* Normal indicates $\mathrm{HbA}_{1 \mathrm{c}}$ levels < 5.7\%; AGR indicates prediabetes and potential DM with $\mathrm{HbA} 1 \mathrm{c}$ levels $\geq 5.7 \%$; $\mathrm{Non}-\mathrm{DM}$ indicates $\mathrm{Hb} \mathrm{A}_{1 \mathrm{c}}$ levels $<6.5 \%$; Potential DM indicates $\mathrm{HbA}_{1 \mathrm{c}}$ levels $\geq 6.5 \%$. ${ }^{* *}$ Sens: sensitivity; Spec: Specificity.

Initially, the validity of the test for identifying AGR was performed using ROC analysis (Tables 3 and 4). First, the AUC was calculated using laboratory FPG $\geq 100 \mathrm{mg} / \mathrm{dl}$ as a diagnosis of AGR (Table 3). According to the evaluation, the performance of RBG cut-off point of $110 \mathrm{mg} / \mathrm{dl}$, RBG cut-off point of $140 \mathrm{mg} / \mathrm{dl}$ and POC $\mathrm{HbA}_{1 \mathrm{c}}$ in predicting FPGdefined AGR was moderate (AUC $=0.65 ; 95 \%$ CI $0.59-0.70,0.69 ; 95 \%$ CI $0.62-0.75$ and 0.72 ; 95\% CI 0.67-0.78, respectively).

Next, ROC analysis to identify subjects with potential type $2 \mathrm{DM}$ was performed using FPG $\geq 126 \mathrm{mg} / \mathrm{dl}$ as a gold standard for the diagnosis of potential type 2 DM (Table 3). Based on the ROC curves, the performances of RBG $\geq 200 \mathrm{mg} / \mathrm{dl}$ with symptoms of $\mathrm{DM}$ and POC $\mathrm{HbA}_{1 \mathrm{c}}$ in predicting FPG-defined type $2 \mathrm{DM}$ was high (AUC = 0.94; 95\% CI 0.86-1.0 and 0.99; 95\% CI 0.98-0.99, respectively).

Subsequently, the validity of the test for identifying AGR was performed using ROC analysis and laboratory-based $\mathrm{HbA}_{1 \mathrm{c}} \geq 5.7 \%$ as a diagnosis of AGR (Table 4 ). The performances of RBG cut-off point of $110 \mathrm{mg} / \mathrm{dl}$, RBG cut-off point of $140 \mathrm{mg} / \mathrm{dl}$, and $\mathrm{POC} \mathrm{HbA}_{1 \mathrm{c}}$ 
in predicting laboratory $\mathrm{HbA}_{1 \mathrm{c}}$-defined AGR were also moderate and comparable to that using laboratory FPG $\geq 100 \mathrm{mg} / \mathrm{dl}$ as a diagnosis of AGR (AUC $=0.63 ; 95 \%$ CI 0.57-0.69, 0.69; 95\% CI 0.62-0.77 and 0.81; 95\% CI 0.78-0.84, respectively).

Finally, the ROC analysis was performed using laboratory-based $\mathrm{HbA}_{1 \mathrm{c}} \geq 6.5 \%$ as a gold standard for the diagnosis of DM (Table 4). Based on the ROC curves the performances of RBG $\geq 200 \mathrm{mg} / \mathrm{dl}$ with symptoms of $\mathrm{DM}$ and $\mathrm{POC} \mathrm{HbA}_{1 \mathrm{c}} \geq 6.5 \%$ in predicting $\mathrm{HbA}_{1 \mathrm{c}}$-defined potential $\mathrm{DM}$ was high (AUC = 0.90; 95\% CI 0.79-1.00 and 0.99; 95\% CI 0.98-0.99, respectively).

\section{Discussion}

In our previous study, dental patients were screened for potential hyperglycemia using POC $\mathrm{HbA}_{1 \mathrm{c}}$ and we found that the prevalence of hyperglycemia defined as POC $\mathrm{HbA}_{1 \mathrm{c}} \geq 5.7 \%$ was $33.8 \%$ [12]. In this study, we re-investigated the prevalence of hyperglycemia and confirmed the prevalence of AGR using hospital-based laboratory methods. It was found that $49 \%$ of subjects had hyperglycemia defined as $\mathrm{POC} \mathrm{HbA}_{1 \mathrm{c}} \geq 5.7 \%$ (Table 2). In addition, the prevalence of subjects with RBG $\geq 110 \mathrm{mg} / \mathrm{dl}$ was as high as $63 \%$. The prevalence was reduced to $32 \%$ when the cut-off level was increased to $\geq 140 \mathrm{mg} / \mathrm{dl}$ and this prevalence was comparable to a study by Jadhav and colleagues who reported the prevalence of hyperglycemia in dental patients to be 35\% using this RBG cut-off level of $140 \mathrm{mg} / \mathrm{dl}$ [18]. The high prevalence in our current study may be due to the fact that the subjects in this study were dental patients who had emergency dental problems and requested emergency dental treatment. It is well established that patients with type $2 \mathrm{DM}$ or prediabetes have a higher risk of periodontal disease and other dental problems $[19,20]$. This might have some influence in causing this high prevalence and implied that a significant portion of patients with dental problems might have undiagnosed hyperglycemia.

When the hospital-based laboratory methods were utilized to reevaluate the prevalence of AGR and potential type $2 \mathrm{DM}$, we found that the prevalence of AGR was $25 \%$ and $17 \%$ when FPG $\geq 100 \mathrm{mg} / \mathrm{dl}$ and laboratory-based $\mathrm{HbA}_{1 \mathrm{c}} \geq 5.7 \%$ were used, respectively (Table 2). The prevalence of $25 \%$ and $17 \%$ were higher to the estimated prevalence of hyperglycemia in the Thai population (13\%) [14]. When the prevalence of potential type $2 \mathrm{DM}$ was considered, this prevalence was $4 \%$ in both measurements using the cut-off levels of FPG $\geq 126 \mathrm{mg} / \mathrm{dl}$ and $\mathrm{HbA}_{1 \mathrm{c}} \geq 6.5 \%$. This prevalence was higher than that reported in the Thai population by Aekplakorn and colleagues as well $(2.3 \%$ defined as FPG $\geq 126 \mathrm{mg} / \mathrm{dl}$ ). As stated before, the subjects who came to receive dental treatment might have an underlying hyperglycemic condition. Therefore, screening of hyperglycemia in a dental setting using POC methods is worth conducting, and we may be able to detect a significant portion of patients with undiagnosed type $2 \mathrm{DM}$.

To investigate the performance of POC $\mathrm{HbA}_{1 \mathrm{c}}$ in identifying subjects with AGR, the sensitivities and specificities between the POC $\mathrm{HbA}_{1 \mathrm{c}}$ and hospital-based laboratory methods, including FPG and laboratory-based $\mathrm{HbA}_{1 \mathrm{c}}$ were analyzed. The sensitivity was $82 \%$, and the specificity was $62 \%$ when the POC $\mathrm{HbA}_{1 \mathrm{c}} \geq 5.7 \%$ was compared to FPG $\geq 100 \mathrm{mg} / \mathrm{dl}$ (Table 3). The sensitivity increased to $100 \%$ when POC $\mathrm{HbA}_{1 \mathrm{c}} \geq 5.7 \%$ was compared to laboratory-based $\mathrm{HbA}_{1 \mathrm{c}} \geq 5.7 \%$ (Table 4) but the specificity was similar to that compared to laboratory FPG (62\%). In terms of screening for potential type $2 \mathrm{DM}$, the sensitivity and specificity were $100 \%$ and $97 \%$ compared to both measurements using FPG $\geq 126 \mathrm{mg} / \mathrm{dl}$ and $\mathrm{HbA}_{1 \mathrm{c}} \geq 6.5 \%$ (Tables 3 and 4). This result indicates that screening for AGR and potential type $2 \mathrm{DM}$ using $\mathrm{POC} \mathrm{HbA}_{1 \mathrm{c}}$ is possible, with moderate to high sensitivity and specificity compared to the standardized methods, and this screening method should be encouraged in the dental setting.

Comparing our result to a previous study by Genco and colleagues conducted in the U.S.A., 1022 dental patients were screened for hyperglycemia. Of these, $416(41 \%)$ had $\mathrm{HbA}_{1 \mathrm{c}} \geq 5.7 \%$ and were referred to see their physicians and $35 \%$ of these subjects received a final diagnosis from their physicians within 1 year. The diagnoses were type $2 \mathrm{DM}(12 \%)$, high risk of developing type $2 \mathrm{DM}(23 \%)$, and no type $2 \mathrm{DM}(64 \%)$ [21]. Recently in 2019, 
changes in screening practices for prediabetes and type $2 \mathrm{DM}$ since the recommendation for $\mathrm{HbA}_{1 \mathrm{c}}$ testing have been reported and encouraged [22]. One study recruited 12,772 eligible patients and reported that when these patients diagnosed with hyperglycemia from the first screening were followed, only $26 \%$ of patients screened with blood glucose levels as opposed to $36 \%$ of patients screened with $\mathrm{HbA}_{1 \mathrm{c}}$ were diagnosed to have continuous hyperglycemia. Hence this result encouraged the use of $\mathrm{POC} \mathrm{HbA}_{1 \mathrm{c}}$ as a screening tool for referral or follow-up of patients with hyperglycemia.

The reason that we preferred RBG was because of the availability for a dental professional to possess a glucometer. According to Tables 3 and 4, the RBG with different cut-off levels ( $\geq 110 \mathrm{mg} / \mathrm{dl}$ or $\geq 140 \mathrm{mg} / \mathrm{dl}$ ) were used for screening of hyperglycemia compared to laboratory FPG and $\mathrm{HbA}_{1 \mathrm{c}}$. The level of $\mathrm{RBG} \geq 110 \mathrm{mg} / \mathrm{dl}$ was used since it was recommended in a study by Herman et al. that this level of RBG exhibited good sensitivity in identifying people with previously undiagnosed DM regardless of age and time since last food or drink [13]. It was demonstrated that when using the cut-off level of RBG $\geq 110 \mathrm{mg} / \mathrm{dl}$, the sensitivity was high compared to laboratory FPG (85\%) (Table 3) and $\mathrm{HbA}_{1 \mathrm{c}}(85 \%)$ (Table 4), however, the specificity was quite low (approximately $40 \%$ compared to both techniques) (Tables 3 and 4). When the levels of RBG $\geq 140 \mathrm{mg} / \mathrm{dl}$ was used, it was obvious that the sensitivities were reduced to $60 \%$ and $64 \%$ and the specificities were increased to $77 \%$ and $74 \%$ compared to FPG and laboratory-based $\mathrm{HbA}_{1 \mathrm{c}}$, respectively (Tables 3 and 4 ). When the levels of RBG were used for the screening of potential type $2 \mathrm{DM}$ (POC RBG $\geq 200 \mathrm{mg} / \mathrm{dl}$ ), the sensitivities and specificities were very high (more than $80 \%$ ). A major objective of most screening tests is to reduce morbidity or mortality in the population group being screened for the disease by early detection [23]. Although the result suggested that RBG might be more specific in identifying dental patients with potential type $2 \mathrm{DM}$, we still believe that the RBG could give strong benefits for the screening of dental patients with AGR. Our result also suggested that the cut-off level of RBG $\geq 110 \mathrm{mg} / \mathrm{dl}$ could be used with high sensitivity to be able to screen more patients who might have AGR.

According to Tables 3 and 4 , the performances of $\mathrm{RBG}$ and $\mathrm{POC} \mathrm{HbA}_{1 \mathrm{c}}$ in predicting FPG-defined AGR were evaluated. Based on the ROC curves, all methods exhibited moderate performance in predicting patients with AGR when FPG was used for the diagnosis. If the laboratory-based $\mathrm{HbA}_{1 \mathrm{c}}$ was to be used as a diagnosis of having $\mathrm{AGR}$, the performances of RBG and POC $\mathrm{HbA}_{1 \mathrm{c}}$ in predicting laboratory-based $\mathrm{HbA}_{1 \mathrm{c}}$-defined AGR were moderate as well. These performances in predicting patients with potential type $2 \mathrm{DM}$ were increased compared to the performances in predicting patients with AGR. This result suggested that using these POC methods in screening dental patients with undiagnosed type $2 \mathrm{DM}$ might exhibit higher validity than in screening patients with AGR.

In a previous population-based survey of 795 people aged 35-60 in rural areas of Uganda, Mayega et al. determined FPG using glucometer and POC $\mathrm{HbA}_{1 \mathrm{c}}$ using $\mathrm{A}_{1 \mathrm{cNow}}{ }^{\circledR}$ kit and evaluated the relationship between FPG-defined DM status and POC $\mathrm{HbA}_{1 \mathrm{c}}$ values [9]. From their study, with FPG as the reference, an agreement between FPG and POC $\mathrm{HbA}_{1 \mathrm{c}}$ in classifying undiagnosed type $2 \mathrm{DM}$, was moderate $(\mathrm{AUC}=0.75)$. It is possible that the performance in our study was high since our subjects were dental patients and the subjects in the study by Mayega et al. were the general population. Since the method to identify dental patients with hyperglycemia was only retrieving a drop of blood, we assumed that screening of dental patients with hyperglycemia is beneficial thus that early diagnosis of patients with hyperglycemia can be recognized and may result in early referral and prevention of patients from having complications from chronic hyperglycemia.

One of the limitations in this study might be the low number of subjects identified to have undiagnosed type $2 \mathrm{DM}$. More subjects may be needed in future studies thus that more patients with undiagnosed type $2 \mathrm{DM}$ will be identified. Moreover, we screened subjects with anemia using only the questionnaire and not the laboratory measurement. Since the measurement of POC $\mathrm{HbA}_{1 \mathrm{c}}$ using the DCA Vantage ${ }^{\circledR}$ analyzer can be affected by abnormal red blood cells, undiagnosed anemia might have influenced the prevalence 
of hyperglycemia in this study. Despite these limitations, we found that $\mathrm{POC} \mathrm{HbA}_{1 \mathrm{c}}$ and RBG had the potential ability to identify dental patients with undiagnosed type 2 DM and, to some extent, for AGR. It is well-established that early detection and appropriate metabolic management of affected individuals can significantly delay the development of most complications. This will provide dental professionals with a tool to directly involve themselves in the healthcare of the patients seen in the dental clinic, particularly in the identification of patients with undiagnosed type 2 DM.

\section{Conclusions}

The results from this study indicated that POC testing, including $\mathrm{POC} \mathrm{HbA}_{1 \mathrm{c}}$ and RGB, could be used as a potential tool for screening of subjects with potential type 2 DM and AGR in a dental setting.

Author Contributions: Conceptualization, S.-o.P.K., C.T. and W.K.; methodology, S.-o.P.K., S.T. and W.K.; subject recruitment, S.-o.P.K., M.S. and T.T.; data analysis, M.S. and C.T.; investigation, M.S. and T.T.; resources, S.-o.P.K. and M.S.; writing—original draft preparation, S.-o.P.K. and M.S.; writingreview and editing, S.-o.P.K., W.K., C.T. and P.F.; funding acquisition, S.-o.P.K. and M.S. All authors have read and agreed to the published version of the manuscript.

Funding: This research received no external funding.

Institutional Review Board Statement: The study was conducted according to the guidelines of the Declaration of Helsinki, and approved by the Institutional Review Board (or Ethics Committee) of the Faculty of Dentistry/Faculty of Pharmacy, Mahidol University (MU-DT/PY IRB 2017/047.2308).

Informed Consent Statement: Informed consent was obtained from all subjects involved in the study.

Data Availability Statement: The data presented in this study are available on request from the corresponding author. The data are not publicly available due to ethical issue.

Acknowledgments: The authors would like to thank the Primary and Emergency Unit, Faculty of Dentistry, Mahidol University for all the supports and the Faculty of Tropical Medicine, Mahidol University regarding laboratory investigation.

Conflicts of Interest: The authors declare no conflict of interest.

\section{References}

1. American Diabetes Association. 2. Classification and Diagnosis of Diabetes: Standards of Medical Care in Diabetes-2018. Diabetes Care 2018, 41, S13-S27. [CrossRef]

2. Saudek, C.D.; Herman, W.H.; Sacks, D.B.; Bergenstal, R.M.; Edelman, D.; Davidson, M.B. A new look at screening and diagnosing diabetes mellitus. J. Clin. Endocrinol. Metab. 2008, 93, 2447-2453. [CrossRef]

3. Aekplakorn, W.; Chariyalertsak, S.; Kessomboon, P.; Assanangkornchai, S.; Taneepanichskul, S.; Putwatana, P. Prevalence of Diabetes and Relationship with Socioeconomic Status in the Thai Population: National Health Examination Survey, 2004-2014. J. Diabetes Res. 2018, 2018, 1654530. [CrossRef]

4. Rohlfing, C.L.; Wiedmeyer, H.M.; Little, R.R.; England, J.D.; Tennill, A.; Goldstein, D.E. Defining the relationship between plasma glucose and $\mathrm{HbA}(1 \mathrm{c})$ : Analysis of glucose profiles and $\mathrm{HbA}(1 \mathrm{c})$ in the Diabetes Control and Complications Trial. Diabetes Care 2002, 25, 275-278. [CrossRef]

5. Rahbar, S.; Blumenfeld, O.; Ranney, H.M. Studies of an unusual hemoglobin in patients with diabetes mellitus. Biochem. Biophys. Res. Commun. 1969, 36, 838-843. [CrossRef]

6. World Health Organization. Use of Glycated Haemoglobin (Hba1c) in the Diagnosis of Diabetes Mellitus: Abbreviated Report of A WHO Consultation; World Health Organization: Geneva, Swizerland, 2011.

7. Kennedy, L.; Herman, W.H.; Team, G.A.C.S. Glycated hemoglobin assessment in clinical practice: Comparison of the A1cNow point-of-care device with central laboratory testing (GOAL A1C Study). Diabetes Technol. Ther. 2005, 7, 907-912. [CrossRef] [PubMed]

8. Wang, Y.; Peng, W.; Tang, J.; Dong, L.; Gu, C.; Zhang, X.; Zhou, J.; Jia, W. Verification of a novel point-of-care HbA $1 \mathrm{c}$ device in real world clinical practice by comparison to three high performance liquid chromatography instruments. Biochem. Med. 2018, 28, 020705. [CrossRef] [PubMed]

9. Mayega, R.W.; Guwatudde, D.; Makumbi, F.E.; Nakwagala, F.N.; Peterson, S.; Tomson, G.; Ostenson, C.G. Comparison of fasting plasma glucose and haemoglobin A1c point-of-care tests in screening for diabetes and abnormal glucose regulation in a rural low income setting. Diabetes Res. Clin. Pract. 2014, 104, 112-120. [CrossRef] [PubMed] 
10. Albisser, A.M.; Sakkal, S.; Wright, C. Home blood glucose prediction: Validation, safety, and efficacy testing in clinical diabetes. Diabetes Technol. Ther. 2005, 7, 487-496. [CrossRef] [PubMed]

11. Glurich, I.; Bartkowiak, B.; Berg, R.L.; Acharya, A. Screening for dysglycaemia in dental primary care practice settings: Systematic review of the evidence. Int. Dent. J. 2018, 68, 369-377. [CrossRef] [PubMed]

12. Tantipoj, C.; Sakoolnamarka, S.S.; Supa-amornkul, S.; Lohsoonthorn, V.; Deerochanawong, C.; Khovidhunkit, S.P.; Hiransuthikul, N. Screening for Type 2 Diabetes Mellitus and Prediabetes Using Point-of-Care Testing for Hba1c among Thai Dental Patients. Southeast. Asian J. Trop. Med. Public Health 2017, 48, 455-465.

13. Herman, W.H.; Taylor, G.W.; Jacobson, J.J.; Burke, R.; Brown, M.B. Screening for prediabetes and type 2 diabetes in dental offices. J. Public Health Dent. 2015, 75, 175-182. [CrossRef]

14. Aekplakorn, W.; Chariyalertsak, S.; Kessomboon, P.; Sangthong, R.; Inthawong, R.; Putwatana, P.; Taneepanichskul, S.; Thai National Health Examination Survey, I.V.S.G. Prevalence and management of diabetes and metabolic risk factors in Thai adults: The Thai National Health Examination Survey IV, 2009. Diabetes Care 2011, 34, 1980-1985. [CrossRef]

15. Fleiss, J.L.; Tytun, A.; Ury, H.K. A simple approximation for calculating sample sizes for comparing independent proportions. Biometrics 1980, 36, 343-346. [CrossRef] [PubMed]

16. WHO Expert Consultation. Appropriate body-mass index for Asian populations and its implications for policy and intervention strategies. Lancet 2004, 363, 157-163. [CrossRef]

17. Engelgau, M.M.; Thompson, T.J.; Smith, P.J.; Herman, W.H.; Aubert, R.E.; Gunter, E.W.; Wetterhall, S.F.; Sous, E.S.; Ali, M.A. Screening for diabetes mellitus in adults. The utility of random capillary blood glucose measurements. Diabetes Care 1995, 18, 463-466. [CrossRef]

18. Jadhav, A.N.; Tarte, P.R.; Puri, S.K. Dental clinic: Potential source of high-risk screening for prediabetes and type 2 diabetes. Indian J. Dent. Res. 2019, 30, 851-854. [CrossRef] [PubMed]

19. Nascimento, G.G.; Leite, F.R.M.; Vestergaard, P.; Scheutz, F.; Lopez, R. Does diabetes increase the risk of periodontitis? A systematic review and meta-regression analysis of longitudinal prospective studies. Acta Diabetol 2018, 55, 653-667. [CrossRef]

20. Nazir, M.A.; AlGhamdi, L.; AlKadi, M.; AlBeajan, N.; AlRashoudi, L.; AlHussan, M. The burden of Diabetes, Its Oral Complications and Their Prevention and Management. Open Access Maced. J. Med. Sci. 2018, 6, 1545-1553. [CrossRef]

21. Genco, R.J.; Schifferle, R.E.; Dunford, R.G.; Falkner, K.L.; Hsu, W.C.; Balukjian, J. Screening for diabetes mellitus in dental practices: A field trial. J. Am. Dent. Assoc. 2014, 145, 57-64. [CrossRef]

22. Evron, J.M.; Herman, W.H.; McEwen, L.N. Changes in Screening Practices for Prediabetes and Diabetes Since the Recommendation for Hemoglobin A1c Testing. Diabetes Care 2019, 42, 576-584. [CrossRef] [PubMed]

23. Maxim, L.D.; Niebo, R.; Utell, M.J. Screening tests: A review with examples. Inhal. Toxicol. 2014, 26, 811-828. [CrossRef] [PubMed] 